\title{
Exercise pulmonary haemodynamics predict outcome in patients with systemic sclerosis
}

\author{
Adriana Stamm ${ }^{1}$, Stéphanie Saxer ${ }^{1}$, Mona Lichtblau ${ }^{1}$, Elisabeth D. Hasler ${ }^{1}$, \\ Suzana Jordan ${ }^{2}$, Lars C. Huber ${ }^{1}$, Konrad E. Bloch ${ }^{3}$, Oliver Distler ${ }^{2}$ and \\ Silvia Ulrich ${ }^{3}$
}

Affiliations: ${ }^{1}$ Dept of Pulmonology, University Hospital Zurich, Switzerland. ${ }^{2}$ Division of Rheumatology, University Hospital Zurich, Switzerland. ${ }^{3}$ Dept of Pulmonology, University Hospital Zurich and Center for Integrative Human Physiology, University of Zurich, Switzerland.

Correspondence: Silvia Ulrich, Department of Pulmonology, UniversitätsSpital Zürich, Rämistrasse 100, $\mathrm{CH}-8091$ Zürich, Switzerland. E-mail: silvia.ulrichdusz.ch

ABSTRACT The aim of the present study was to investigate the prognostic value of exercise haemodynamics measured during right heart catheterisation (RHC) in patients with systemic sclerosis (SSc) referred for evaluation of pulmonary hypertension.

SSc patients undergoing RHC at rest and during maximal supine incremental cycle exercise were grouped into resting precapillary pulmonary hypertension (PHrest) (mean pulmonary artery pressure (mPAP) $\geqslant 25 \mathrm{mmHg}$, pulmonary artery wedge pressure $<15 \mathrm{mmHg}$ ), exercise-induced pulmonary hypertension (PHex) (mPAP $\geqslant 30 \mathrm{mmHg}$ and $\mathrm{mPAP} / \mathrm{cardiac}$ output $>3 \mathrm{mmHg} \cdot \mathrm{L}^{-1} \cdot \mathrm{min}^{-1}$ at maximal exercise), and without pulmonary hypertension (PHnone). Patients' characteristics, haemodynamics and follow up data were compared between groups.

72 SSc patients were followed for median (interquartile range) 33 (15-55) months. Mean (95\% CI) survival without transplantation estimated by Kaplan-Meyer analysis was $4.4(0.8-2.9)$ years in PHrest $(\mathrm{n}=17), 5.2$ (4.4-6.1) years in PHex $(\mathrm{n}=28)$ and 9.5(8.4-10.6) years in PHnone ( $\mathrm{n}=27 ; \mathrm{p}<0.05$ versus others). In Cox regression models, the exercise-induced increase in mPAP (hazard ratio (HR) 1.097, 95\% CI $1.002-1.200$ ) and the coefficient of pulmonary vascular distensibility alpha (HR 0.100 , 95\% CI $0.012-$ 0.871 ) controlled for age, but not resting haemodynamics predicted transplant-free survival.

Among SSc patients with normal mPAP at rest, an excessive increase in mPAP during exercise and an impaired vascular distensibility may indicate an early stage of pulmonary vasculopathy, associated with reduced survival similar to resting pulmonary hypertension patients.

@ERSpublications

Exercise pulmonary haemodynamics may add important information for management of patients with systemic sclerosis http://ow.ly/Jqkw3034lqp

Editorial comment in: Eur Respir J 2016; 48: 1549-1552.

Received: May 182016 | Accepted after revision: Aug 032016 | First published online: Oct 062016

Support statement: This work was supported by grants from the Swiss National Science Foundation and the Zurich Lung League. Funding information for this article has been deposited with the Open Funder Registry.

Conflict of interest: Disclosures can be found alongside this article at erj.ersjournals.com

Copyright OERS 2016 


\section{Introduction}

Pulmonary arterial hypertension (PAH) is a common and severe complication in patients with connective tissue disease $[1,2]$. In particular, PAH is most frequent in systemic sclerosis (SSc) with an estimated prevalence of $7.5-12 \%$ [3-5] and it is the leading cause of death in SSc with almost one-third of SSc-related deaths [6-9].

The gold standard for diagnosis of PAH in SSc patients is right heart catheterisation (RHC) and PAH is defined as precapillary pulmonary hypertension with a mean pulmonary artery pressure (mPAP) $\geqslant 25 \mathrm{mmHg}$ with a pulmonary artery wedge pressure (PAWP) $\leqslant 15 \mathrm{mmHg}$ at rest [10]. Exercise pulmonary hypertension, defined by $\mathrm{mPAP}>30 \mathrm{mmHg}$ during exercise, has been eliminated as a diagnostic criterion for pulmonary hypertension due to lack of agreement on a cut-off value for exercise pulmonary hypertension, with insufficient supporting data in the literature and missing specification of this criterion on the exact exercise level, type and posture [11]. While mPAP at rest has been found to be age-independent in healthy individuals, mPAP during exercise appeared to frequently exceed $30 \mathrm{mmHg}$ at high cardiac output (CO) during exercise, especially in individuals aged $\geqslant 50$ years [12]. Nevertheless, an exercise-induced increase in mPAP will most probably be a precursor of resting pulmonary hypertension in patients at risk, such as in those with SSc. Thus, more research is needed to define the clinical relevance and outcome of patients with exercise-induced pulmonary hypertension in this patient population [13-15]. Recently, Hervé et al. [16] found that a combined maximum mPAP $>30 \mathrm{mmHg}$ and total pulmonary vascular resistance (TPVR) $>3 \mathrm{WU}$ during exercise was associated with pulmonary vascular disease independent of age, sex, body mass index and diagnosis.

As PAH associated with SSc is a prevalent and potentially fatal condition for which treatment is available, it is important to identify those SSc patients who are at high risk of having early pulmonary vascular disease with the intent to delay or even prevent deterioration. The aim of this study was therefore to review exercise RHC performed at our centre in SSc patients and to study the prognostic value of exercise pulmonary haemodynamics in terms of transplant-free survival and to compare it with established markers of disease severity.

\section{Methods}

\section{Study design and patients}

Data on all SSc patients who had diagnostic RHC at our pulmonary hypertension-referral centre due to clinical suspicion of pulmonary hypertension (exertional dyspnoea and/or unclearly reduced diffusing capacity of the lung for carbon monoxide (DLCO) or a forced vital capacity (FVC)/DLCO >1.6) were reviewed. It has been our clinical practice since September 2005 to perform supine incremental cycle ergometry during RHC in all patients with precapillary pulmonary hypertension unless they are unstable or suffer from musculoskeletal problems precluding cycling. SSc patients were censored in November 2015. Patients with postcapillary pulmonary hypertension, defined as PAWP $>15 \mathrm{mmHg}$ at rest or $>20 \mathrm{mmHg}$ during exercise, and patients with other pulmonary hypertension classification (FVC $<60 \%$ predicted or other aetiology of pulmonary hypertension than SSc) were excluded from the study [17].

Available chest computed tomography was reviewed and scored for the percentage of lung volume with fibrosis with 0:none, 1: <20\%; and 2: $>20 \%$. Demographics, clinical classification of SSc as limited cutaneous SSc (lcSSc), diffuse cutaneous SSc (dcSSc) or not classified SSc, WHO functional class (WHOFC), 6-min walk distance (6MWD), pulmonary function, echocardiography and venous and arterial blood test results were recorded [18]. Follow-up data including WHO-FC, 6MWD, haemodynamics by echocardiography and $\mathrm{N}$-terminal pro-brain natriuretic peptide (NT-proBNP) from the regular visits every 3-6 months up to 2 years after the RHC were analysed until occurrence of an event (defined as death or lung transplantation) or until November 2015 in censored patients. PAH-specific medication consisting of endothelin-receptor antagonists (ERA) or phosphodiesterase-5-inhibitors (PDE-5-inhibitors) given during follow-up for $>3$ months was noted. All patients gave their written informed consent to have their data registered and the study was approved by the local ethical review board (KEK 2012-0125).

\section{Exercise RHC}

A balloon-tipped, triple-lumen, fluid-filled 7.5 Fr Swan Ganz catheter (Baxter/Edwards, Deerfield, IL, USA) was introduced via an internal jugular vein [19]. Transducers were set at the mid-axillary line and zeroed to atmospheric pressure [20,21]. Cardiac output was assessed by continuous thermodilution (Baxter/Edwards) and cardiac output/body surface area yielded the cardiac index (CI). The pulmonary vascular resistance (PVR) and systemic vascular resistance (SVR) were calculated as: $P V R=(m P A P$ $-\mathrm{PAWP}) /$ cardiac output; $\mathrm{SVR}=(\mathrm{mPAP}-\mathrm{RAP}) /$ cardiac output, the transpulmonary gradient $(\mathrm{TPG})=\mathrm{mPAP}$ -PAWP. Mean baseline measurements after $15 \mathrm{~min}$ of rest were noted as resting values. Supine cycling exercise was performed according a symptom-limited, stepwise incremental protocol starting with 10 Watt followed by increase of 10 Watt every $3 \mathrm{~min}$ (TheraVital, Medica GmbH, Ravensburg) at a cycle rate of 
60 revolutions $\cdot \min ^{-1}$. Measurements were taken during the last $30 \mathrm{~s}$ of each step. Pulmonary artery distensibility index $\alpha$ was calculated as previously described $[19,22]$.

SSc patients were allocated to three different groups according to their resting and exercise haemodynamics: 1) resting precapillary pulmonary hypertension ( $\mathrm{mPAP} \geqslant 25 \mathrm{mmHg}$, PAWP $\leqslant 15 \mathrm{mmHg}$ ) (PHrest); 2) exercise precapillary pulmonary hypertension (mPAP at maximal exercise $>30 \mathrm{mmHg}$ with a $\mathrm{mPAP} / \mathrm{cardiac}$ output $>3 \mathrm{mmHg} \cdot \mathrm{min} \cdot \mathrm{L}^{-1}$, PAWP $<20 \mathrm{mmHg}$ ) (PHex) [16]; and 3) patients without pulmonary hypertension (PHnone).

\section{Statistical analysis}

Data are summarised by medians (interquartile range) for continuous values in order to account for some non-normally distributed values and frequencies (\%) for binary and categorical variables. Baseline variables by groups were compared using Mann-Whitney U-test or Fisher's exact test when appropriate. Follow-up comparisons were calculated using the Wilcoxon test. Transplant-free survival was calculated by Kaplan-Meier analysis with comparisons of the time to event performed by log-rank test. Cox regression was used for predictors of transplant-free survival. A two-sided p-value $<0.05$ was considered as significant throughout. SPSS 22 (SPSS, Chicago, IL, USA) was used.

\section{Results}

\section{Baseline characteristics}

From 84 exercise-RHC performed in SSc, 12 patients had postcapillary pulmonary hypertension at rest or exercise and were excluded (figure 1). From the 72 eligible patients, 17 (24\%) had PHrest, 28 (39\%) had PHex, 27 (38\%) had PHnone. Demographic and clinical data by haemodynamic groups are shown in table 1. The majority was female with lcSSc, history of Raynaud, an impaired DLCO and anti-centromere antibodies-positive, irrespective of the subgroup. At baseline, patients with PHrest were significantly older and had a worse WHO-FC than PHex and PHnone (table 1). The 6MWD was significantly shorter in patients with PHrest (394 (324-478) m) compared with PHex (489 (357-565) m) ( $\mathrm{p=0.030)}$ and PHnone (492 (365-558) m) $(\mathrm{p}=0.040)$. There was no difference in FVC \% predicted/DLCO \% predicted ratio amongst the groups, although PHrest tended to have a higher ratio. Five out of 11 with PHrest had pulmonary fibrosis (four out of five $<20 \%$ ), four out of 19 with PHex had fibrosis (three out of four $<20 \%$ ) and three out of 17 with PHnone had fibrosis (two out of three $<20 \%$ ). Serum analysis showed higher levels of NT-proBNP for PHrest compared with PHex and PHnone. Targeted treatment for PAH was introduced in all patients with PHrest and off-label by judgments of the treating physicians in 16 (57\%) patients with PHex and consisted of ERA and/or PDE-5-inhibitors.

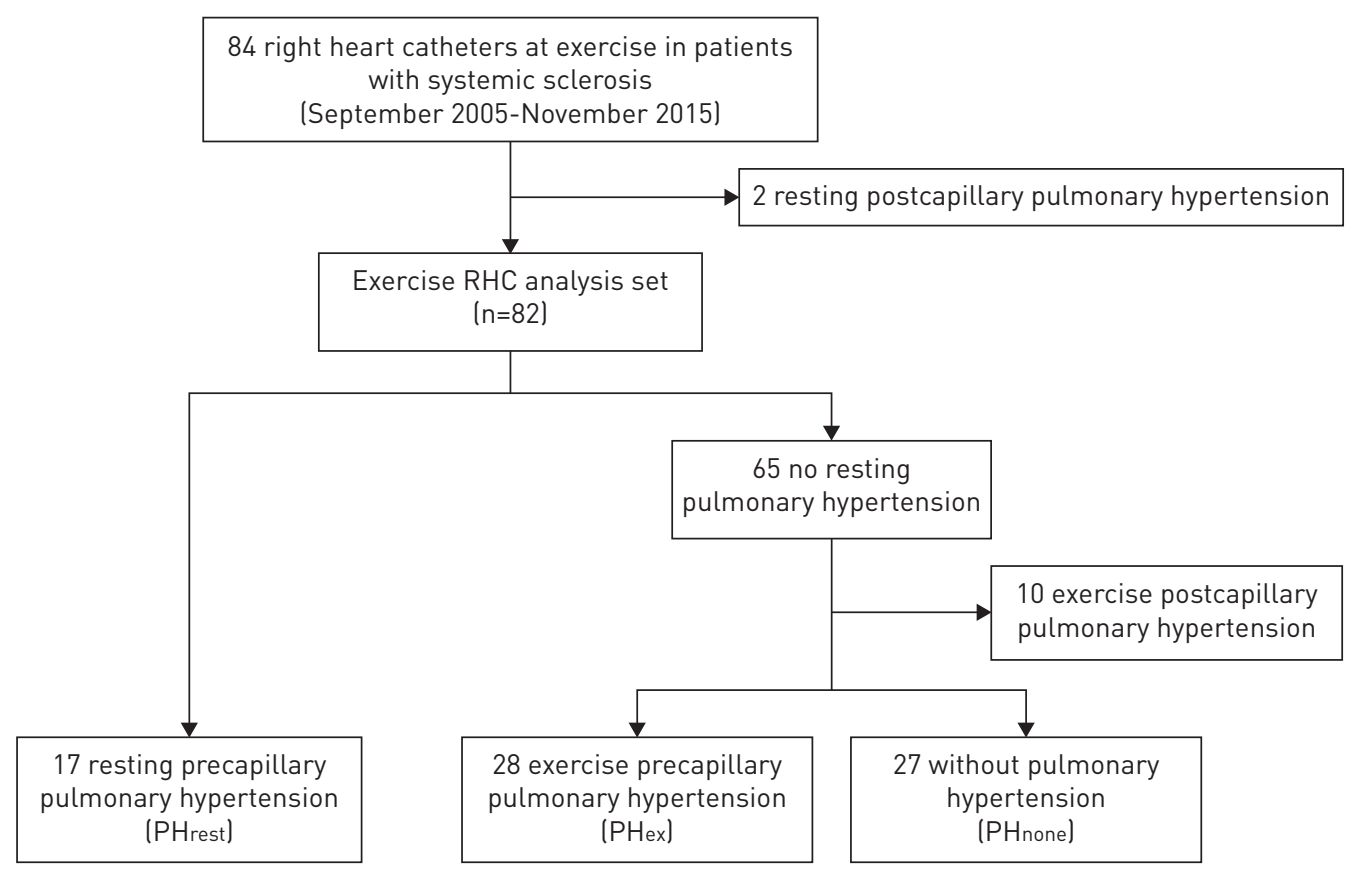

FIGURE 1 The haemodynamic classification of 84 patients with systemic sclerosis who were investigated with exercise right heart catheterisation are shown. $\mathrm{RHC}$ : right heart catheter; $\mathrm{PH}$ : pulmonary hypertension. 
TABLE 1 Characteristics of systemic sclerosis patients according to their haemodynamic classification into resting pulmonary hypertension (PHrest), pulmonary hypertension during exercise ( $\mathrm{PHex}$ ) and without pulmonary hypertension ( $\mathrm{PHnone}$ )

\begin{tabular}{|c|c|c|c|}
\hline \multirow[t]{2}{*}{ Variable } & \multicolumn{3}{|c|}{ Patient groups } \\
\hline & PHrest & PHex & PHnone \\
\hline Patients & $17(20)$ & $28(33)$ & $27(32)$ \\
\hline \multicolumn{4}{|l|}{ Demographical parameters } \\
\hline Female & $12(71)^{*}$ & $24(86)$ & $26(94)$ \\
\hline Age years & $66(59-70) *$ & $63(54-74)^{\text {กิก }}$ & $51(42-65)$ \\
\hline \multicolumn{4}{|l|}{ Clinical parameters } \\
\hline WHO-FC & $3(2-3)^{* *}, \# \#$ & 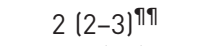 & $2(1-2)$ \\
\hline$|/| I$ & $5(29)^{*, \# \#}$ & $17(61)$ & $21(77)$ \\
\hline III/IV & $12(71)$ & 10 (39) & 5 (19) \\
\hline BMI $\mathrm{kg} \cdot \mathrm{m}^{-2}$ & $24.5(20.3-27.2)$ & $25.4(23.4-28.2)$ & $23.1(19.9-27.2)$ \\
\hline \multicolumn{4}{|l|}{ Exercise capacity } \\
\hline $6 \mathrm{MWD} \mathrm{m}$ & $394(324-478)^{*, \# \#}$ & 489 (357-565) & 492 (365-558) \\
\hline $\mathrm{SpO}_{2}$ after $6 \mathrm{MWD} \%$ & $94(87-96)$ & $94(87-96)^{\text {ตा }}$ & $96(92-98)$ \\
\hline Heart rate after 6 MWD beats. $\min ^{-1}$ & 106 (92-135) & $110(98-125)$ & $116(100-135)$ \\
\hline \multicolumn{4}{|l|}{ Pulmonary function } \\
\hline $\mathrm{FEV}_{1} \%$ predicted & $77(68-87)$ & $84(67-101)$ & $93(81-105)$ \\
\hline TLC \% predicted & $87(79-100)$ & 97 (83-109) & $97(83-108)$ \\
\hline FVC $\%$ predicted & $80(69-91)^{*}, \# \#$ & $93(83-110)$ & $96(83-106)$ \\
\hline DLco \% predicted & $38(35-68)$ & $59(45-63)$ & $59(50-65)$ \\
\hline FVC $\%$ predicted/DLco $\%$ predicted & $2.0(1.3-2.5)$ & $1.7(1.5-2)$ & $1.6(1.4-1.9)$ \\
\hline \multicolumn{4}{|l|}{ Blood analysis } \\
\hline Antinuclear anti-body positive & $17(100)$ & $28(100)$ & $27(100)$ \\
\hline Anti-Scl-70 positive & $2(12)$ & $4(28)^{\text {กาก }}$ & $11(41)$ \\
\hline Anti-centromere positive & $7(41)$ & 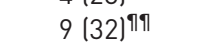 & 10 (37) \\
\hline NT-proBNP ng $\cdot L^{-1}$ & $500(276-1360)^{*, \# \#}$ & $153(74-428)$ & $132(68-362)$ \\
\hline \multicolumn{4}{|l|}{ Echocardiography } \\
\hline Left ventricular ejection fraction $\%$ & $63(60-69)$ & $63(62-65)$ & $62(58-65)$ \\
\hline Right atrium area $\mathrm{cm}^{2}$ & $20(15-24)^{*, \# \#}$ & $16(15-17)$ & $14(13-18)$ \\
\hline Tricuspid regurgitation velocity $\mathrm{m} \cdot \mathrm{s}^{-1}$ & $3.2(2.8-3.9)^{* *}$,\#\# & $2.6(2.5-2.9)$ & $2.2(2.2-2.9)$ \\
\hline \multicolumn{4}{|l|}{ Treatment } \\
\hline Targeted treatment for pulmonary hypertension & $17(100)$ & $16(57)$ & $0(0)$ \\
\hline
\end{tabular}

Data are presented as $\mathrm{n}(\%)$ or median (interquartile range). WHO-FC: World Health Organization functional class; BMI: body mass index; 6MWD: 6-min walk distance; $\mathrm{SpO}_{2}$ : arterial oxygen saturation measured by pulse oximetry; FEV1: forced expiratory volume in $1 \mathrm{~s}$; TLC: total lung capacity; FVC: forced vital capacity; $D$ Lco: diffusing capacity of the lung for carbon monoxide; NT-proBNP: $N$-terminal-pro brain natriuretic peptide. *: $p<0.05$ PHrest to PHnone; ${ }^{* *}$ : $p<0.001$ PHrest to PHnone; ${ }^{\# \#}$ : $p<0.001$ PHrest to PHex; ${ }^{\text {१ा: }}$ p $<0.001$ PHex to PHnone.

\section{Haemodynamics}

Per definition, patients with PHrest and PHex had higher mPAP, TPG and PVR at rest and during exercise compared with $\mathrm{PH}_{\text {none }}(\mathrm{p}<0.001$ in all comparisons; table 2$)$. In PHrest, median mPAP was 30 (26-43) $\mathrm{mmHg}$ at rest and $50(45-62) \mathrm{mmHg}$ at maximal workload, whereas in PHex mPAP was significantly lower at rest $(20(17-21)) \mathrm{mmHg}$ and increased to $33(31-36) \mathrm{mmHg}(\mathrm{p}=0.000)$. Patients with PHrest achieved a lower maximal workload than patients with $\mathrm{PHnone}$, the difference to PHex was not significant. Increase of mPAP during exercise was greater in PHrest with 17 (11-23) $\mathrm{mmHg}$ and PHex with 14 (13-16) $\mathrm{mmHg}$ compared with PHnone $7(4.5 ; 9) \mathrm{mmHg}(\mathrm{p}=0.000)$. The pressure-flow ratio (mPAP/cardiac output) was lowest in PHnone (3.7 (1.7-7.9)), intermediate in PHex (8.2 (6.1-30)) and highest in PHrest (24 $(12.6-82.2)($ all $\mathrm{p}<0.05)$. Arterial oxygen saturation $\left(\mathrm{SaO}_{2}\right)$ was significantly lower in PHrest compared with PHex $(\mathrm{p}=0.009)$ and PHnone.

\section{Functional assessments during follow-up}

All patients who received target treatment for PAH were followed up after 3, 12 and 24 months (table 3). Over the observation period, the number of treated patients with WHO-FC III or IV decreased in PHrest from $12(71 \%)$ at baseline to two (40\%) after 24 months and in treated PHex from six (38\%) to one (17\%). Physical performance assessed by 6MWD improved in PHrest from 394 (324-478) $\mathrm{m}$ to 476 (370-593) $\mathrm{m}$ and in PHex with PAH target treatment from 506 (357-570) $\mathrm{m}$ to 520 (386-560) $\mathrm{m}$ (not significant). 
TABLE 2 Haemodynamics of systemic sclerosis patients according to their haemodynamic classification into resting pulmonary hypertension (PHrest), pulmonary hypertension during exercise (PHex) and without pulmonary hypertension (PHnone)

\begin{tabular}{|c|c|c|c|}
\hline \multirow[t]{2}{*}{ Variable } & \multicolumn{3}{|c|}{ Patient groups } \\
\hline & PHrest & PHex & PHnone \\
\hline \multicolumn{4}{|l|}{ Resting haemodynamics } \\
\hline HR beats $\cdot \min ^{-1}$ & 79 (69-93) & $72(63-78)$ & $75(68-81)$ \\
\hline $\mathrm{mBP} \mathrm{mmHg}$ & $101(89-113)^{*, \#}$ & 89 (81-105) & 89 (82-99) \\
\hline mPAP $\mathrm{mmHg}$ & $30(26-43)^{* *}$ & $20(17-21)^{\text {กิก }}$ & $16(14-18)$ \\
\hline PAWP mmHg & $12(10-13)^{*, \#}$ & $10(8-12)$ & $9(7-11)$ \\
\hline RAP $\mathrm{mmHg}$ & $8(5-9)^{*, \#}$ & $8(4-8)$ & $5(4-8)$ \\
\hline TPG mmHg & $20(16-25)^{* *, \# \#}$ & $9(8-12)^{\pi}$ & $7(6-8)$ \\
\hline $\mathrm{CI} \mathrm{L} \cdot \mathrm{min}^{-1} \cdot \mathrm{m}^{-2}$ & $2.9(2.3-3.6)$ & $3.3(2.7-3.9)$ & $3.2(2.8-3.6)$ \\
\hline $\mathrm{CO} L \cdot \min ^{-1}$ & $5.1(3.7-6.4)$ & $5.6(4.4-6.9)$ & $5.3(4.4-6.7)$ \\
\hline PVR WU & 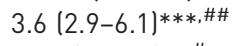 & $1.6(1.3-2.2)^{\pi}$ & $1.3(0.9-1.7)$ \\
\hline $\mathrm{SaO}_{2} \%$ & $91(89-94)^{* *, \#}$ & 95 (92-95) & $95(93-96)$ \\
\hline $\mathrm{SvO}_{2} \%$ & $68(62-70)^{*, \#}$ & $71(67-72)$ & $71(69-74)$ \\
\hline \multicolumn{4}{|l|}{ Exercise haemodynamics } \\
\hline Maximal workload W & $20(15-30) *$ & $30(20-40)$ & $30(30-40)$ \\
\hline Maximal HR beats $\cdot \min ^{-1}$ & $111(101-126)$ & 111 (99-123) & $104(100-120)$ \\
\hline Maximal mBP mmHg & $110(99-127)^{*}$ & $111(97-118)^{\pi}$ & 99 (89-109) \\
\hline Maximal mPAP mmHg & $50(45-62)^{* *, \# \#}$ & $33(31-36)^{\text {กิก }}$ & $24(21-25)$ \\
\hline Maximal PAWP mmHg & $15(11-17)^{*}$ & $16(14-19)^{119}$ & $11(7-14)$ \\
\hline Maximal RAP mmHg & $10(8-18)^{* *}, \#$ & $8(5-10)^{9}$ & $5(2-6)$ \\
\hline Maximal TPG mmHg & $34(26-44)^{* *, \# \#}$ & $19(16-21)^{\text {ติก }}$ & $12(10-16)$ \\
\hline Maximal $\mathrm{Cl} \mathrm{L} \cdot \mathrm{min}^{-1} \cdot \mathrm{m}^{-2}$ & $3.5(2.6-4.3)$ & $4.2(3.6-4.8)$ & $3.9(3.5-4.8)$ \\
\hline Maximal CO L. $\min ^{-1}$ & $6.2(4.1-7.4)^{\#}$ & $7.1(6.6-8.1)$ & $6.9(5.8-8.9)$ \\
\hline Maximal PVR WU & $5.4(3.8-10)^{* *}, \# \#$ & $2.7(2-3.1)^{n}$ & $1.7(1.0-2.1)$ \\
\hline Maximal $\mathrm{SaO}_{2} \%$ & $91(84-95)^{* *, \#}$ & $95(89-96)$ & $96(95-97)$ \\
\hline Maximal $\mathrm{SvO}_{2} \%$ & $43(36-54)$ & $47(42-54)$ & $49(44-55)$ \\
\hline$\Delta \mathrm{mPAP} / \Delta \mathrm{CO} \mathrm{mmHg} \cdot \mathrm{min} \cdot \mathrm{L}^{-1}$ & $24(15-81)^{* *, \#}$ & $9.4(6-27)^{\text {ติศ }}$ & $3.7(2-7)$ \\
\hline Increase of mPAP with exercise $\mathrm{mmHg}$ & $18(12-24)^{* *}$ & $14(13-16)^{\text {กิก }}$ & $7(5-9)$ \\
\hline Increase mPAP per Watt $\mathrm{mmHg} \cdot \mathrm{W}^{-1}$ & $0.8(0.5-1.2)^{* *, \#}$ & $0.6(0.3-0.8)^{\text {ติก }}$ & $0.2(0.1-0.3)$ \\
\hline Distensibility index $\alpha \% \cdot \mathrm{mmHg}^{-1}$ & $0.5(0.2-0.6)^{* *}, \# \#$ & $0.7(0.6-0.8)^{\text {กาก }}$ & $1.2(0.9-1.6)$ \\
\hline \multicolumn{4}{|c|}{ 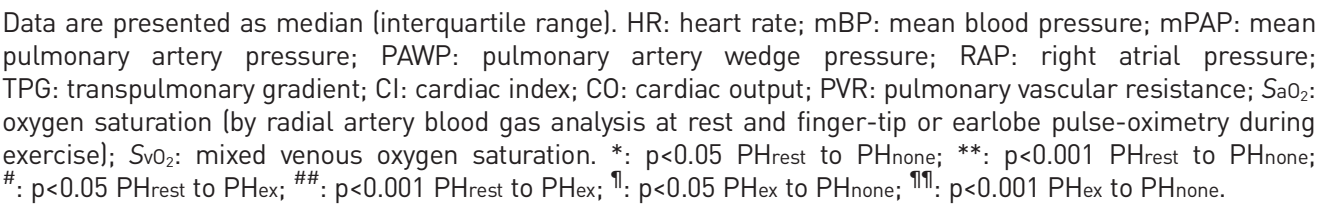 } \\
\hline
\end{tabular}

TABLE 3 Follow-up of patients who received pulmonary arterial hypertension targeted medical therapy after 3,12 and 24 months

\begin{tabular}{|c|c|c|c|c|c|c|}
\hline & \multicolumn{3}{|c|}{ PHrest } & \multicolumn{3}{|c|}{ PHex with treatment } \\
\hline WHO-FC & $2(2-3)$ & $3(2-3)$ & $2(2-3)$ & $2(2-3)$ & $2(2-2)$ & $2(2-3)$ \\
\hline $1 / I I$ & $5(29)$ & $6(43)$ & $4(67)$ & $10(63)$ & $11(79)$ & $7(64)$ \\
\hline III/IV & $12(71)$ & $8(57)$ & 2 (33) & $6(38)$ & $3(21)$ & $4(36)$ \\
\hline 6MWD & $394(324-478)$ & $456(331-480)$ & $464(416-595)$ & $506(357-570)$ & $517(386-563)$ & $517(364-630)$ \\
\hline $\mathrm{SpO}_{2}$ after $6 \mathrm{MWD} \%$ & $94(87-96)$ & $87(86-95)$ & $90(89-96)$ & $93(87-96)$ & 95 (94-97) & $92(85-97)$ \\
\hline Tricuspid regurgitation velocity $\mathrm{m} \cdot \mathrm{s}^{-1}$ & $3.2(2.8-3.9)$ & & $3(2.9-3.9)$ & 2. $(2.5-2.8)$ & & $2.6(2.5-2.7)$ \\
\hline Right atrium area $\mathrm{cm}^{2}$ & $18(17-21)$ & & $20(16-22)$ & $15(13-17)$ & & $16(13-19)$ \\
\hline
\end{tabular}

Data are presented as median (interquartile range) or $\mathrm{n}$ (\%). WHO-FC: World Health Organization functional class; 6MWD: 6-min walk distance; $\mathrm{SpO}_{2}$ : arterial oxygen saturation measured by pulse oximetry; HR: heart rate; NT-proBNP: N-terminal pro-brain natriuretic peptide. 


\section{Survival}

Ten patients died during the median follow-up period of 33 (13-55) months, three (18\%) with PHrest, five (18\%) with PHex and two (7\%) with PHnone. Death was related to progressive right heart failure in seven patients (three in PHrest, four in PHex), to respiratory tract infections in one patient (PHnone), to multiorgan disease with microangiopathy of the kidney in one patient (PHnone) and to a psychiatric disorder in one patient (PHex). In three out of four PHex patients who died with right heart failure, 2.8-2.6 years after diagnosis, progression to resting pulmonary hypertension could be documented by echocardiography 12 22 months after the RHC. One patient with progressive PHex and one with PHrest underwent lung transplantation. The 1-, 3- and 5-year event-free survivals were $88 \%, 82 \%$ and $77 \%$ for PHrest, $100 \%, 93 \%$ and $82 \%$ for $\mathrm{PHex}$ and $100 \%, 93 \%$ and $93 \%$ for PHnone, respectively. Mean (95\% CI) survival without transplantation by Kaplan-Meyer was $4.4(0.8-2.9)$ years in PHrest $(\mathrm{n}=17), 5.2$ (4.4-6.1) years in PHex $(\mathrm{n}=28)$ and 9.5 (8.4-10.6) years in PHnone and significantly better in PHnone versus PHrest $(\mathrm{p}=0.019)$ or $\mathrm{PH}_{\mathrm{ex}}(\mathrm{p}=0.043$ ) (figure 2). No difference in transplant-free survival could be found in PHrest versus $\mathrm{PHex}$ or patients with $\mathrm{PH}$ ex who were treated versus untreated. Univariate cox-regression revealed WHO-FC, NT-proBNP blood levels, FVC/DLCO ratio, mPAP at maximal exercise or increase during exercise, increase mPAP per Watt, pulmonary vascular distensibility index $\alpha$, resting mixed-venous oxygen saturation $\left(\mathrm{SvO}_{2}\right)$ and oxyhaemoglobin as predictors of transplant-free survival in the SSc-patient collective (table 4). Resting haemodynamics did not significantly predict survival. Multivariate analysis controlled for age revealed that mPAP increase during exercise (hazard ratio 1.097, 95\% CI 1.002-1.200), mPAP increase per Watt (hazard ratio $8.131,95 \%$ CI 2.209-29.928) and distensibility index $\alpha$ (hazard ratio $0.100,95 \%$ CI $0.012-0.871$ ) remained predictors of transplant free survival.

\section{Discussion}

In this study, we showed for the first time that SSc patients with $\mathrm{PHex}$ had worse transplant-free survival compared with SSc without pulmonary hypertension. The reduced transplant-free survival of SSc with PHex was similar to that of SSc patients with PHrest (mPAP $\geqslant 25 \mathrm{mmHg}$, PAWP $\leqslant 15 \mathrm{mmHg}$ ). Transplant-free survival could be predicted by the maximal mPAP and increase in mPAP with exercise, but not by resting haemodynamics.

The diagnostic and prognostic value of pulmonary haemodynamics during exercise has been debated for a long time $[12,14,23]$. Recently, Hervé et al. [16] suggested that the combination of the two criteria, mPAP at maximal exercise $>30 \mathrm{mmHg}$ and TPVR $>3 \mathrm{WU}$ during exercise, identified patients who were shown to suffer from pulmonary vascular disease due to chronic thromboembolism or other aetiology according to subsequent clinical and/or histological evaluation. This approach including an exercise related, flow-dependent criterion retained sensitivity at 0.93 but improved specificity to 1.0 to diagnose pulmonary vascular disease compared to the maximal mPAP or TPVR during exercise. Expanding on these observations, we have recently shown that the pulmonary haemodynamic response to exercise predicts outcome in patients diagnosed with PAH or inoperable chronic thromboembolic pulmonary hypertension [19]. Few studies have investigated exercise haemodynamics by RHC in SSc patients [1, 12, 24, 25] and, to our knowledge, the

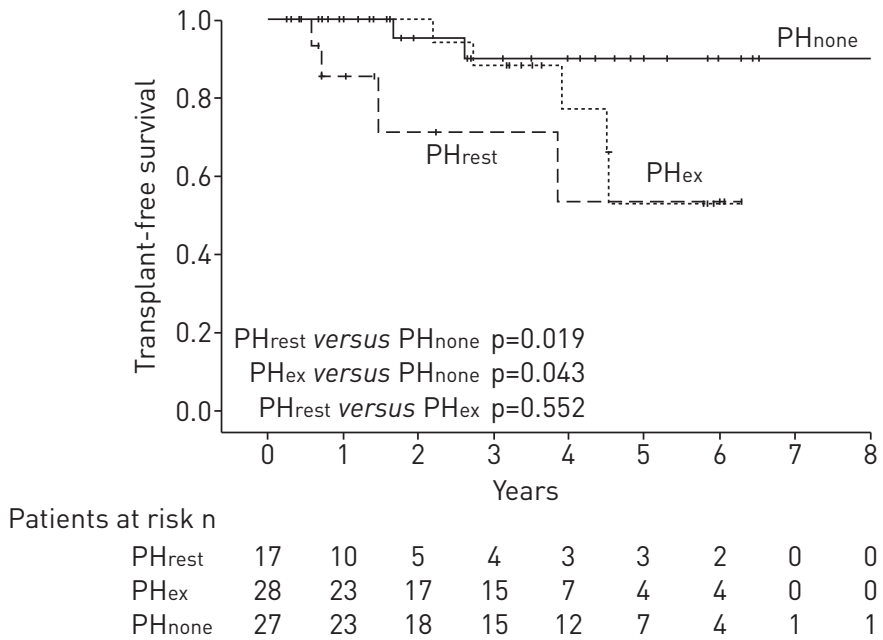

FIGURE 2 Transplant-free survival by Kaplan-Meier method and number at risk of 72 patients according to their haemodynamic classification is shown. Patients were censored November 2015. PHrest: resting precapillary pulmonary hypertension; PHex: exercise precapillary pulmonary hypertension; PHnone: patients without pulmonary hypertension. 
TABLE 4 Univariate Cox-proportional hazards analysis of transplantation or death for functional parameters and haemodynamics at rest and during exercise in 72 systemic sclerosis patients assessed during right heart catheterisation

\begin{tabular}{|c|c|c|}
\hline & Hazard ratio $(95 \% \mathrm{Cl})$ & p-value \\
\hline Age years & $1.032(0.977-1.090)$ & 0.253 \\
\hline WHO-FC & $3.423(1.634-7.171)$ & 0.001 \\
\hline 6MWD m & $0.997(0.993-1.002)$ & 0.241 \\
\hline NT-proBNP $n g \cdot L^{-1}$ & $1.000(1.000-1.001)$ & 0.019 \\
\hline DLCO \% & $0.966(0.921-1.013)$ & 0.151 \\
\hline FVC $\%$ predicted $/ D$ Lco $\%$ predicted & $2.476(1.015-6.041)$ & 0.046 \\
\hline Tricuspid regurgitation velocity $\mathrm{m} \cdot \mathrm{s}^{-1}$ & $1.378(0.565-3.363)$ & 0.481 \\
\hline Right atrium area $\mathrm{cm}^{2}$ & $0.997(0.858-1.159)$ & 0.970 \\
\hline $\mathrm{SaO}_{2} \%$ & $0.788(0.665-0.934)$ & 0.006 \\
\hline $\mathrm{SvO}_{2} \%$ & $0.881(0.807-0.963)$ & 0.005 \\
\hline \multicolumn{3}{|c|}{ Resting haemodynamics by right heart catheterisation } \\
\hline HR beats $\cdot \mathrm{min}^{-1}$ & $0.991(0.938-1.048)$ & 0.760 \\
\hline $\mathrm{mBP} \mathrm{mmHg}$ & $0.998(0.955-1.042)$ & 0.911 \\
\hline mPAP $\mathrm{mmHg}$ & $1.029(0.989-1.070)$ & 0.152 \\
\hline PAWP mmHg & $1.027(0.823-1.280)$ & 0.815 \\
\hline RAP $\mathrm{mmHg}$ & $1.117(0.921-1.355)$ & 0.259 \\
\hline $\mathrm{COL} \cdot \mathrm{min}^{-1}$ & $0.891(0.589-1.350)$ & 0.587 \\
\hline Cardiac index $\mathrm{L} \cdot \mathrm{min}^{-1} \cdot \mathrm{m}^{-2}$ & $0.686(0.307-1.533)$ & 0.358 \\
\hline PVR WU & $1.118(0.957-1.307)$ & 0.161 \\
\hline \multicolumn{3}{|c|}{ Values obtained at maximal supine cycling exercise } \\
\hline Maximal workload W & $0.978(0.930-1.028)$ & 0.384 \\
\hline Maximal HR beats $\cdot \min ^{-1}$ & $1.019(0.981-1.059)$ & 0.333 \\
\hline Maximal mBP mmHg & $1.026(0.984-1.069)$ & 0.229 \\
\hline Maximal mPAP mmHg & $1.039(1.004-1.074)$ & 0.028 \\
\hline Maximal PAWP mmHg & $1.026(0.910-1.160)$ & 0.657 \\
\hline Maximal RAP mmHg & $1.055(0.943-1.180)$ & 0.352 \\
\hline Maximal cardiac index $\mathrm{L} \cdot \mathrm{min}^{-1} \cdot \mathrm{m}^{-2}$ & $0.778(0.431-1.406)$ & 0.406 \\
\hline Maximal CO L. $\min ^{-1}$ & $0.940(0.740-1.254)$ & 0.673 \\
\hline Maximal PVR WU & $1.081(0.977-1.196)$ & 0.131 \\
\hline Maximal $\mathrm{SvO}_{2} \%$ & $0.965(0.900-1.034)$ & 0.309 \\
\hline$\Delta \mathrm{mPAP} / \Delta \mathrm{CO} \mathrm{mmHg} \cdot \mathrm{min} \cdot \mathrm{L}^{-1}$ & $1.015(0.996-1.035)$ & 0.127 \\
\hline Increase mPAP with exercise $\mathrm{mmHg}$ & $1.103(1.015-1.199)$ & 0.021 \\
\hline Increase mPAP/power output mmHg.watt ${ }^{-1}$ & $8.098(2.408-27.236)$ & 0.001 \\
\hline Distensibility index $\alpha \% \cdot \mathrm{mmHg}^{-1}$ & $0.1(0.012-0.865)$ & 0.036 \\
\hline
\end{tabular}

WHO-FC: World Health Organization functional class; 6MWD: 6-min walk distance; NT-proBNP: $N$-terminal pro-brain natriuretic peptide; $D\llcorner C 0$ : diffusing capacity of the lung for carbon monoxide; FVC: forced vital capacity of the lung; $\mathrm{SaO}_{2}$ : oxygen saturation (by radial artery blood gas analysis at rest and finger-tip or earlobe pulse-oximetry during exercise); $\mathrm{SvO}_{2}$ : mixed venous oxygen saturation; HR: heart rate; mBP: mean blood pressure; mPAP: mean pulmonary artery pressure; PAWP: pulmonary artery wedge pressure; RAP: right atrial pressure; TPG: transpulmonary gradient; CO: cardiac output; PVR: pulmonary vascular resistance.

current study is the first investigating the prognostic relevance of PHex in SSc patients diagnosed by the combined pressure and flow-related criteria.

In this cohort, SSc patients with PHex who did not fulfill criteria for pulmonary hypertension, had an $\mathrm{mPAP}$ and PVR at rest that was slightly higher compared with patients without pulmonary hypertension, thus their resting haemodynamics profile was intermediate between PHrest and PHnone (table 2). This intermediate haemodynamic profile of SSc patients with PHex persisted during exercise (table 2). These findings support the notion suggested previously $[12,26]$ that $\mathrm{PHex}$ may be a pathophysiological stage intermediate between normal haemodynamics and pulmonary vasculopathy with pulmonary hypertension at rest. In line with these assumptions, Conduiffe et al. [1] found that $19 \%$ of patients with PHex progressed to PHrest after a mean time of 2 years and 108 days. We observed that patients with PHex were older, showed higher WHO-FC, higher serum levels of NT-proBNP and lower $\mathrm{SpO}_{2}$ after 6MWD compared with SSc patients without pulmonary hypertension, all consistent with a more advanced clinical state. Hence, including exercise haemodynamics into the evaluation of SSc patients might help to identify those patients with pulmonary vascular disease who are at risk of developing PHrest and who might possibly benefit from 
early therapy. In the current study, the PHrest patients had relatively low PVR compared with the published cohort, potentially related to early pulmonary hypertension diagnosis resulting from the yearly screening programme for SSc patients with assessment of increasing dyspnoea and decreasing exercise capacity and DLCO [27]. The comparably low percentage of postcapillary pulmonary hypertension in our cohort is due to our practice not to perform exercise RHC in patients with clinically clear resting postcapillary pulmonary hypertension. In our SSc cohort, the maximal workload achieved during exercise RHC was relatively low, especially in patients without pulmonary hypertension, potentially due to a combination of supine exercise with a 3-min step duration and/or lack of training in this chronically ill SSc population. Some patients without pulmonary hypertension revealed a comparatively high $\mathrm{mPAP} /$ cardiac output slope, mainly due to an inadequate increase in cardiac output despite a maximal mPAP well below $30 \mathrm{mmHg}$. During follow-up of these patients over 0.5-6.5 years, none developed PAH but three out of five developed left heart disease. PHrest patients had higher PAWP at rest and PHrest and PHex patients had higher PAWP at maximal exercise compared with patients without pulmonary hypertension. Although none of the PHex patients had a PAWP > $20 \mathrm{mmHg}$ at maximal exercise, we cannot exclude a concurrent latent left heart disease with diastolic dysfunction in some of these elderly SSc patients.

Some studies reported that in patients with PAH associated with SSc, DLCO was impaired, sometimes for many years prior to the development of PAH $[3,25,28,29]$. Also an increase in FVC/DLCO was found to be associated with increased risk for PAH [29] and was used as a variable in a two-step detection programme for PAH associated with SSc [30]. In our cohort, DLCO and FVC/DLCO ratio were similar between groups, most probably related to the fact that these parameters are part of our selection criteria to perform RHC in SSc patients and thus, our PHnone group might had a relatively low DLCO due to selection bias. Another promising investigation to differentiate SSc patients with pulmonary vasculopathy from those not affected might be cardiopulmonary exercise testing [31], which was unfortunately not available in our cohort.

\section{Survival}

The diagnosis of PHex in SSc patients is relevant and associated with a worse transplant-free survival similar to that in PHrest. This finding indicates that $\mathrm{PHex}$ is a sign of early, albeit prognostically relevant, pulmonary vascular disease in SSc patients known to be at risk of developing PAH. As this is not a prospective study, we do not have regular follow-up haemodynamics of all patients with $\mathrm{PHex}$ and thus do not know the percentage of patients who subsequently developed PAH. A hint towards a progressive nature of PHex from our study is the fact that four out of five patients with $\mathrm{PHex}$ died of progressive right heart failure. Whether early detection of pulmonary vascular disease in SSc patients and early treatment would increase transplant-free survival is still not clear. In the past years, prospective cohort studies using various approaches to the early detection of pulmonary vascular disease in SSc have been performed $[9,25,30]$. However, there is still no consensus about the best screening programmes, indicating the need for further studies.

It has been demonstrated that SSc patients with $\mathrm{PAH}$, despite more favourable haemodynamics in RHC and comparable therapy, are more likely to die than patients with idiopathic $\mathrm{PAH}$ [8, 32-34]. Although survival improved in idiopathic PAH in the modern treatment era since 2002 when oral agents for PAH-specific treatment became available, it is controversial whether survival in SSc-associated PAH similarly improved [1, $33,35]$. The worse response to targeted therapy in SSc compared with idiopathic PAH may suggest that idiopathic and SSc-associated PAH have different pathophysiological mechanisms and/or that increased comorbidities in SSc contribute to an unfavourable therapeutic response [36, 37]. Whereas in idiopathic $\mathrm{PAH}$ a homogenous pattern of plexogenic arteriopathy is common, findings in SSc-PAH seem to be more heterogeneous with a remarkable proportion of fibrosis of pulmonary veins and venules, resembling a veno-occlusive disease. This may explain why SSc patients with $\mathrm{PAH}$ are less responsive to treatment compared with idiopathic PAH and have worse outcome [36]. In the present cohort, we found no difference in transplant-survival for $\mathrm{PH}$ ex who received $\mathrm{PAH}$-targeted therapy versus those who did not. As this is not a prospective randomised trial, it is not possible to conclude on the efficacy of PAH therapy in this subgroup.

In our cohort, survival was predicted by mPAP at maximal exercise and increase in mPAP during exercise in addition to baseline WHO-FC, resting $\mathrm{SaO}_{2}$ and $\mathrm{S}_{\mathrm{vO}_{2}}$. Resting haemodynamics, on the other hand, did not predict survival emphasising the additional prognostic value of exercise compared with resting haemodynamics and other established clinical markers of disease severity in PAH.

\section{Follow-up data of SSc patients who received PAH targeted therapy}

All patients with PHrest and 16 (57\%) patients of $\mathrm{PHex}$ were treated with $\mathrm{PAH}$-targeted therapy. During follow-up under therapy after 3,12 and 24 months, 6MWD, serum NT-proBNP and haemodynamics by echocardiography did not change. However, compared with idiopathic PAH [10, 38], the role of these markers to predict disease severity and outcome in SSc is less clear, as they might be influenced by comorbidities $[7,32,39,40]$. As treatment in the current study was not randomised, no firm conclusions 
on efficacy are possible. In addition, we did not do a comparative standardised follow-up assessment of functional class and 6MWD in PHex patients who did not receive PAH targeted therapy. Thus, randomised-controlled trials should elucidate the effectiveness of PAH targeted therapy in SSc patients with early pulmonary vascular disease manifesting as $\mathrm{PHex}$.

\section{Conclusions}

This study indicates that PHex in SSc patients at risk for PAH represents an early form of pulmonary vascular disease. The fact that survival is reduced in SSc patients with PHex compared with PHnone supports the value of assessing pulmonary haemodynamics not only at rest, but also during exercise in these patients. The impaired outcome in SSc with $\mathrm{PH}_{\mathrm{ex}}$ and the frequent disease progression to PHrest not only emphasise the need for development of early pulmonary hypertension detection strategies in SSc, but also suggest that research should be dedicated to answer the question whether initiation of early PAH-targeted medical therapy would improve outcome in this devastating disease.

\section{Acknowledgements}

Author contributions: All authors contributed significantly to the submitted work. A. Stamm, K.E. Bloch and S. Ulrich contributed to the conception and design of the study, the collection, analysis and interpretation of data, and the writing of the manuscript. S. Ulrich is the guarantor of the paper. O. Distler and S. Jordan contributed to data collection and critically revised the manuscript. S. Saxer, E.D. Hasler, M. Lichtblau, L.C. Huber, O. Distler and K.E. Bloch critically revised the manuscript. All authors gave their final approval.

\section{References}

1 Condliffe R, Kiely DG, Peacock AJ, et al. Connective tissue disease-associated pulmonary arterial hypertension in the modern treatment era. Am J Respir Crit Care Med 2009; 179: 151-157.

2 Tyndall AJ, Bannert B, Vonk M, et al. Causes and risk factors for death in systemic sclerosis: a study from the EULAR Scleroderma Trials and Research (EUSTAR) database. Ann Rheum Dis 2010; 69: 1809-1815.

3 Hachulla E, Gressin V, Guillevin L, et al. Early detection of pulmonary arterial hypertension in systemic sclerosis: a French nationwide prospective multicenter study. Arthritis Rheum 2005; 52: 3792-3800.

4 Mukerjee D, St George D, Coleiro B, et al. Prevalence and outcome in systemic sclerosis associated pulmonary arterial hypertension: application of a registry approach. Ann Rheum Dis 2003; 62: 1088-1093.

5 Vonk MC, Broers B, Heijdra YF, et al. Systemic sclerosis and its pulmonary complications in The Netherlands: an epidemiological study. Ann Rheum Dis 2009; 68: 961-965.

6 Steen VD, Medsger TA. Changes in causes of death in systemic sclerosis, 1972-2002. Ann Rheum Dis 2007; 66: 940-944.

7 McLaughlin V, Humbert M, Coghlan G, et al. Pulmonary arterial hypertension: the most devastating vascular complication of systemic sclerosis. Rheumatology (Oxford) 2009; 48: Suppl. 3, iii25-iii31.

8 Kawut SM, Taichman DB, Archer-Chicko CL, et al. Hemodynamics and survival in patients with pulmonary arterial hypertension related to systemic sclerosis. Chest 2003; 123: 344-350.

9 Hachulla E, Carpentier P, Gressin V, et al. Risk factors for death and the 3-year survival of patients with systemic sclerosis: the French ItinérAIR-Sclérodermie study. Rheumatology (Oxford) 2009; 48: 304-308.

10 Hoeper MM, Bogaard HJ, Condliffe R, et al. Definitions and diagnosis of pulmonary hypertension. J Am Coll Cardiol 2013; 62: Suppl. 25, D42-D50.

11 Badesch DB, Champion HC, Sanchez MA, et al. Diagnosis and assessment of pulmonary arterial hypertension. J Am Coll Cardiol 2009; 54: Suppl. 1, S55-S66.

12 Kovacs G, Berghold A, Scheidl S, et al. Pulmonary arterial pressure during rest and exercise in healthy subjects: a systematic review. Eur Respir J 2009; 34: 888-894.

13 Naeije R, Vonk Noordegraaf A, Kovacs G. Exercise-induced pulmonary hypertension: at last! Eur Respir J 2015; 46: 583-586.

14 Naeije R, Vanderpool R, Dhakal BP, et al. Exercise-induced pulmonary hypertension: physiological basis and methodological concerns. Am J Respir Crit Care Med 2013; 187: 576-583.

15 Kovacs G, Olschewski H. Borderline pulmonary pressures in scleroderma - a 'pre-pulmonary arterial hypertension' condition? Arthritis Res Ther 2015; 17: 123.

16 Hervé P, Lau EM, Sitbon O, et al. Criteria for diagnosis of exercise pulmonary hypertension. Eur Respir J 2015; 46: 728-737.

17 Galie N, Humbert M, Vachiery JL, et al. 2015 ESC/ERS Guidelines for the diagnosis and treatment of pulmonary hypertension: The Joint Task Force for the Diagnosis and Treatment of Pulmonary Hypertension of the European Society of Cardiology (ESC) and the European Respiratory Society (ERS): Endorsed by: Association for European Paediatric and Congenital Cardiology (AEPC), International Society for Heart and Lung Transplantation (ISHLT). Eur Respir J 2015; 46: 903-975.

18 van den Hoogen F, Khanna D, Fransen J, et al. 2013 classification criteria for systemic sclerosis: an American College of Rheumatology/European League against Rheumatism collaborative initiative. Arthritis Rheum 2013; 65: 2737-2747.

19 Hasler ED, Müller-Mottet S, Furian M, et al. Pressure-flow during exercise catheterization predicts survival in pulmonary hypertension. Chest 2016; 150: 57-67.

20 Kovacs G, Avian A, Olschewski A, et al. Zero reference level for right heart catheterisation. Eur Respir J 2013; 42: $1586-1594$.

21 Kovacs G, Avian A, Pienn M, et al. Reading pulmonary vascular pressure tracings. How to handle the problems of zero leveling and respiratory swings. Am J Respir Crit Care Med 2014; 190: 252-257.

22 Reeves JT, Linehan JH, Stenmark KR. Distensibility of the normal human lung circulation during exercise. Am J Physiol Lung Cell Mol Physiol 2005; 288: L419-L425. 
23 Simonneau G, Robbins IM, Beghetti M, et al. Updated clinical classification of pulmonary hypertension. J Am Coll Cardiol 2009; 54: Suppl. 1, S43-S54.

24 Saggar R, Khanna D, Furst DE, et al. Exercise-induced pulmonary hypertension associated with systemic sclerosis: four distinct entities. Arthritis Rheum 2010; 62: 3741-3750.

25 Steen V, Chou M, Shanmugam V, et al. Exercise-induced pulmonary arterial hypertension in patients with systemic sclerosis. Chest 2008; 134: 146-151.

26 Tolle JJ, Waxman AB, Van Horn TL, et al. Exercise-induced pulmonary arterial hypertension. Circulation 2008; 118: 2183-2189.

27 Visovatti SH, Distler O, Coghlan JG, et al. Borderline pulmonary arterial pressure in systemic sclerosis patients: a post-hoc analysis of the DETECT study. Arthritis Res Ther 2014; 16: 493.

28 Hachulla E, de Groote P, Gressin V, et al. The three-year incidence of pulmonary arterial hypertension associated with systemic sclerosis in a multicenter nationwide longitudinal study in France. Arthritis Rheum 2009; 60: 1831-1839.

29 Steen V, Medsger TA Jr. Predictors of isolated pulmonary hypertension in patients with systemic sclerosis and limited cutaneous involvement. Arthritis Rheum 2003; 48: 516-522.

30 Coghlan JG, Denton CP, Grünig E, et al. Evidence-based detection of pulmonary arterial hypertension in systemic sclerosis: the DETECT study. Ann Rheum Dis 2014; 73: 1340-1349.

31 Ninaber MK, Hamersma WB, Schouffoer AA, et al. Detection of pulmonary vasculopathy by novel analysis of oxygen uptake in patients with systemic sclerosis: association with pulmonary arterial pressures. Clin Exp Rheumatol 2014; 32: 6 Suppl. 86, S-60-7.

32 Fisher MR, Mathai SC, Champion HC, et al. Clinical differences between idiopathic and scleroderma-related pulmonary hypertension. Arthritis Rheum 2006; 54: 3043-3050.

33 Rubenfire M, Huffman MD, Krishnan S, et al. Survival in systemic sclerosis with pulmonary arterial hypertension has not improved in the modern era. Chest 2013; 144: 1282-1290.

34 Condliffe R, Howard LS. Connective tissue disease-associated pulmonary arterial hypertension. F1000Prime Rep 2015; 7: 06 .

35 Williams $\mathrm{MH}$, Das C, Handler CE, et al. Systemic sclerosis associated pulmonary hypertension: improved survival in the current era. Heart 2006; 92: 926-932.

36 Dorfmuller P, Humbert M, Perros F, et al. Fibrous remodeling of the pulmonary venous system in pulmonary arterial hypertension associated with connective tissue diseases. Hum Pathol 2007; 38: 893-902.

37 Overbeek MJ, Vonk MC, Boonstra A, et al. Pulmonary arterial hypertension in limited cutaneous systemic sclerosis: a distinctive vasculopathy. Eur Respir J 2009; 34: 371-379.

38 McLaughlin VV, Archer SL, Badesch DB, et al. ACCF/AHA 2009 expert consensus document on pulmonary hypertension a report of the American College of Cardiology Foundation Task Force on Expert Consensus Documents and the American Heart Association developed in collaboration with the American College of Chest Physicians; American Thoracic Society, Inc.; and the Pulmonary Hypertension Association. J Am Coll Cardiol 2009; 53: 1573-1619.

39 Le Pavec J, Humbert M, Mouthon L, et al. Systemic sclerosis-associated pulmonary arterial hypertension. Am J Respir Crit Care Med 2010; 181: 1285-1293.

40 Garin MC, Highland KB, Silver RM, et al. Limitations to the 6-minute walk test in interstitial lung disease and pulmonary hypertension in scleroderma. J Rheumatol 2009; 36: 330-336. 